\title{
RELACION ENTRE CARACTERISTICAS DEL TRACTO DIGESTIVOY LOS HABITOS ALIMENTARIOS DE PECES DEL RIOYUCAO, SISTEMA DEL RIO META (COLOMBIA)
}

\section{RELATIONSHIP BETWEEN DIGESTIVE TRACT CHARACTERISTICS AND DI- ETS OF FISHES FROM YUCAO RIVER, META RIVER SYSTEM (COLOMBIA)}

\author{
René M. Sánchez ${ }^{1}$, Germán Galvis² \& Pedro F. Victoriano ${ }^{3}$ \\ ${ }^{1}$ Programa Doctorado en Ciencias Biológicas-Zoología. Universidad de Concepción. Casilla 160-C. Concepción. Chile. \\ E-mail: resanche@udec.cl \\ ${ }^{2}$ Departamento Biología. Fac. de Cs. Universidad Nacional de Colombia. Bogotá. \\ ${ }^{3}$ Depto. Zoología. Fac. de Cs. Naturales y Oceanográficas. Universidad de Concepción. Casilla 160-C. Concepción. Chile.
}

\begin{abstract}
RESUMEN
Se analizó los contenidos estomacales de 21 especies de peces capturadas en el río Yucao, en dos sitios y periodos anuales diferentes. Las estructuras del tracto digestivo fueron clasificadas de acuerdo a la posición de la boca, forma del estómago y dientes, longitud del intestino y número de braquispinas y ciegos pilóricos. Estas características se relacionaron con cuatro hábitos alimentarios generalizados: detritívoros, herbívoros, consumidores de perifiton (perifitófagos) y omnívoros. La forma de los dientes y del estómago fueron las estructuras del digestivo que, en los análisis de correspondencia, se relacionaron mejor con los diferentes tipos de alimentación. De acuerdo a la composición espacial y temporal de los contenidos estomacales en cada especie, se puede establecer que los recursos alimentarios aprovechados por estos organismos son variados y pertenecen a diferentes niveles de la trama trófica. Dentro de las especies analizadas predominaron las omnívoras, que consumen indistintamente material vegetal o animal de origen alóctono o del cuerpo de agua.
\end{abstract}

Palabras Claves: Río Yucao, contenido estomacal, morfología del tracto digestivo, peces tropicales.

\section{ABSTRACT}

Stomach contents of 21 species of fishes catched in two sites of Yucao river on different periods of the year were analyzed. Digestive structures were classified according to the mouth position, stomach and teeth form, intestine length and gill rakers number. These characteristics were related with generalized four food habits: detritivorous, herbivorous, periphyton feeders and omnivorous. Teeth and stomach shapes are the digestive structures that best correspond to food kind according to correspondence analysis. On the other hand, stomach contents in each species were variable, they are at different trophic web levels. Omnivorous species that eat vegetable or animal material, show predominance of allochthonous or autochthonous items.

KEYwORDS: Yucao river, stomach contents, digestive tract morphology, tropical fishes.

\section{INTRODUCCION}

El río Yucao es un tributario del río Meta, sistema del río Orinoco, que nace en la altillanura disecada del departamento del Meta, Colombia, y que, de acuerdo a sus características físicas y químicas, se considera de aguas blancas según la clasificación de Sioli (1975). Las bajas concentraciones de nutrientes hace pensar que la producción biológica en este río es baja al estar limitada la productividad primaria. Sin embargo, su composición íctica es importante para la biodiversidad del sistema río Meta (Sánchez et 
al. 1999), ya que representa cerca de un $40 \%$ de las especies registradas en él y un $20 \%$ de las registradas para toda la cuenca del Orinoco Colombiano. Entonces, ¿cómo se mantiene esta alta diversidad de peces en este sistema de baja producción primaria? Es posible pensar que estos peces se mantienen aprovechando cualquier recurso disponible, presentando diferentes hábitos alimentarios. Asimismo, debido a la presencia de un gran número de especies, algunas de éstas pueden presentar comportamientos alimentarios similares, constituyendo gremios desde un punto de vista trófico (Jaksic 2001).

Gran número de especies de peces de agua dulce no tienen un régimen alimentario estricto, dado que los recursos tróficos disponibles pueden variar a lo largo del año, encontrándose un grado importante de generalismo en la dieta (LoweMcConnell 1987; Castro \& Sánchez 1994). Estas variaciones en la oferta alimentaria pueden estar dadas por diferentes aspectos como cambios en el clima, ciclos biológicos, los que pueden incidir en las fluctuaciones de la abundancia del alimento; además, relaciones interespecíficas entre las poblaciones de peces, como la competencia por los posibles ítemes alimentarios, pueden incidir sobre la disponibilidad de estos recursos.

La variabilidad interespecífica de modelos de alimentación en peces, como respuesta al alimento disponible, ha dado como resultado que éstos presenten versatilidad funcional en las estructuras utilizadas en la adquisición de energía, de acuerdo al hábito que han adquirido (Liem 1980). En varios trabajos se ha demostrado que especies con hábitos alimentarios similares tienen una morfología del tracto digestivo semejante (Nikolsky 1963; Lagler et al. 1977; Camargo 1992). El objetivo del presente trabajo es hacer una aproximación acerca de los hábitos alimentarios de peces del río Yucao, a partir del análisis de contenidos estomacales y la descripción del tracto digestivo. De esta forma se pretende determinar si existen especies con hábitos alimentarios similares, y si hay alguna relación de estos hábitos con las características estructurales del tracto digestivo.

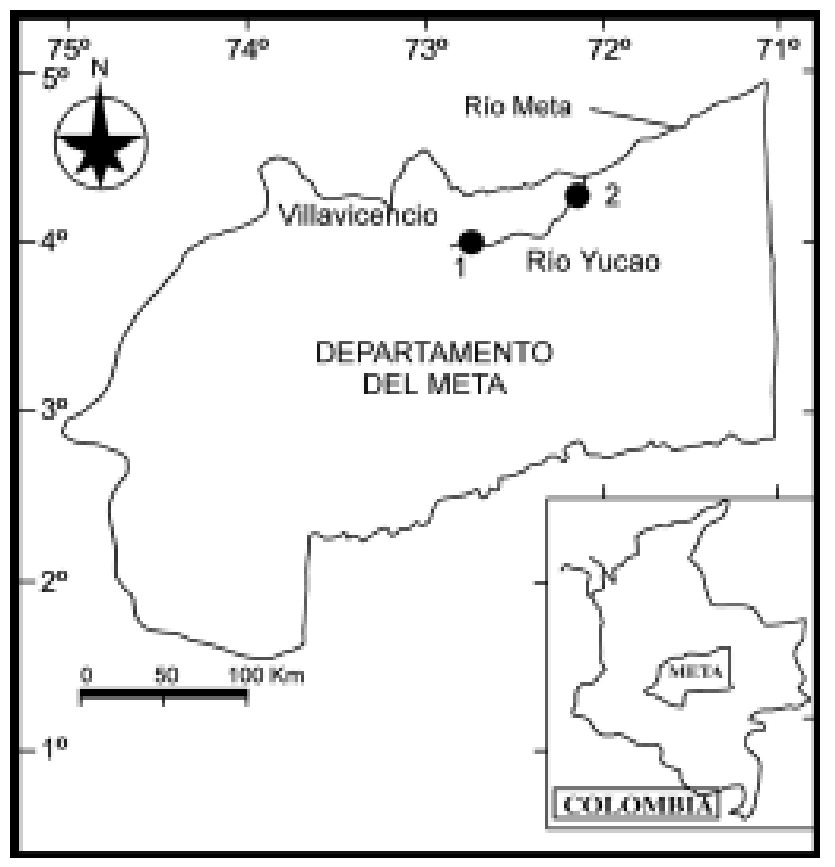

FiguRa 1. Localización geográfica del río Yucao. 1. Sitio de muestreo próximo a su nacimiento, en cercanías al municipio de Puerto López. 2. Sitio de muestreo cerca a su desembocadura en el río Meta, en el municipio de Puerto Gaitán.

Figure 1. Geographic situation of río Yucao. 1. Sample site near to origin, next to Puerto Lopez. 2. Sample site before connection to río Meta, in Puerto Gaitán. 


\section{MATERIALES Y METODOS}

El río Yucao se localiza al norte del departamento del Meta, Colombia, recorriéndolo de oeste a este entre los municipios de Puerto López y Puerto Gaitán (Figura 1). Las capturas de peces se realizaron en la parte alta del río, cerca de su nacimiento (alto Yucao, Punto 1) y en la parte baja, cerca a su desembocadura en el río Meta (bajo Yucao, Punto 2), en dos períodos del año con regímenes lluviosos diferentes, marzo y noviembre. Se utilizaron diferentes artes de pesca como trasmallos de nylon, atarrayas, redes de mano y anzuelos de diferentes tamaños.

A las especies con mayor número de individuos capturados se les registró la longitud estándar, número de braquispinas en el primer arco branquial, posición de la boca, tipo de dentición, forma del estómago, longitud del intestino y presencia de ciegos pilóricos. Esta información fue considerada al momento de relacionar las dietas con características del tracto del digestivo.

Para cada localidad y período del año se obtuvo una muestra de los ítemes consumidos por especie, a partir de la agrupación de los contenidos estomacales de los peces capturados. El volumen total de cada ítem, fue estimado de acuerdo a lo recomendado por Prejs \& Colomine (1981) para casos en que el volumen del contenido estomacal resulta ser muy pequeño. En cada caso se agrupó la muestra por ítem y se cuantificó su volumen total. En aquellas especies en que el contenido estomacal estuvo conformado por detritus se tomaron alícuotas para ser observadas en el microscopio y se cuantificaron por campos. Se realizó una identificación taxonómica de los tipos de presa hasta el nivel taxonómico más bajo posible, con el fin de determinar su procedencia (alóctono o del cuerpo de agua). Para la determinación se utilizaron los trabajos de Forster (1969), Yacubson (1969), Uherkovich \& Schmidt (1974), Pennak (1978), Needham \& Needham (1982) y Roldan (1988).

En el estudio comparativo de las dietas se realizaron análisis de correspondencia (DECORANA) mediante el programa estadístico Golliwog (Wilson 2002), con el fin de detectar la existencia de probables grupos de especies con hábitos tróficos similares. Para estos análisis se consideraron como variables o atributos a las frecuencias de los diferentes ítemes, y los "individuos" correspondieron a las diferentes especies de peces. Con el fin de determinar la eventual existencia de relaciones entre dieta y características estructurales del tracto digestivo, se analizó mediante DECORANA la presencia o ausencia de cada ítem alimentario y cada una de las diferentes morfologías del digestivo.

\section{RESULTADOS}

\section{CARACTERÍSTICAS MORFOLÓGICAS}

La gama de especies comprendió un total de 21 taxa, las que se detallan en la Tabla I. De acuerdo con Gery (1977) y Nelson (1994), éstas pertenecen a nueve familias y cuatro órdenes, lo que se refleja a su vez en la existencia de formas corporales muy variables (Figura 2). Del mismo modo, las estructuras morfológicas relacionadas al sistema digestivo fueron heterogéneas (Tabla II). De acuerdo a la posición de la boca, las especies caracterizadas presentan las siguientes disposiciones: subterminal o ligeramente ubicadas hacia el dorso del animal (p.e. Creagrutus beni; Cyphocharax festivus, Cyphocharax spilurus), terminales (p.e. Bryconops melanurus; Hemigrammus barrigonae) y subinferiores (p.e. Hemiodopsis semitaeniatus; Hemiodus unimaculatus). Los dientes se clasificaron de acuerdo a su forma en caninos, unicúspides, tricúspides, pentacúspides, asimétricos, incisivos, bilobados, cónicos y en algunas especies están ausentes.

El número de braquispinas varió desde 5 para $C$. spilurus, hasta 60 como en $H$. unimaculatus. Hay que tener en cuenta que el tamaño de estas estructuras varía según la distribución en la branquia y también entre especies. Este es el caso de Eigenmannia virescens, que presenta gran número de braquispinas pero de tamaño reducido, mientras que $H$. semitaeniatus presenta un número similar pero de mayor porte.

Los estómagos por su forma se catalogaron como bolsa asimétrica, bilobado, bolsa, alargado y oblongo (Figura 3). El número de ciegos pilóricos fluctuó entre ausentes, (p.e 
TABLA I. Especies, número y rango de talla de individuos utilizados para los análisis de dieta y morfología del tracto digestivo.

TABLE I. Species, sample size and body size range of individuals used for diet analyses and morphology of digestive tracto.

\begin{tabular}{|c|c|c|c|c|c|c|c|}
\hline \multirow{2}{*}{ FAMILIA } & \multirow{2}{*}{ ESPECIE } & \multirow{2}{*}{$\begin{array}{l}\text { NUMERO } \\
\text { INDIVIDUOS }\end{array}$} & \multirow{2}{*}{$\begin{array}{c}\text { LONGITUD ESTANDAR (mm) } \\
\text { Xfl- ds }(\mathrm{m} n-\mathrm{m} / \mathrm{x})\end{array}$} & \multicolumn{4}{|c|}{$\begin{array}{l}\text { SITIO Y EPOCA DE } \\
\text { CAPTURA }\end{array}$} \\
\hline & & & & $\mathrm{AL}$ & AS & $\mathrm{BL}$ & BS \\
\hline \multirow[t]{7}{*}{ Characidae } & Bryconops melanurus (Bloch, 1794) & 37 & $60.1-7.1(41.2-102.1)$ & $\mathrm{X}$ & $\mathrm{X}$ & $\mathrm{X}$ & $\mathrm{X}$ \\
\hline & Creagrutus beni Eigenmann, 1911 & 14 & $49.2-4.6(31.3-64.9)$ & $\mathrm{X}$ & $\mathrm{X}$ & & $\mathrm{X}$ \\
\hline & Hemigrammus barrigonae (Eigenmann y Henn, 1914) & 32 & $33.8-2.6(25.3-40.2)$ & $\mathrm{X}$ & $\mathrm{X}$ & & $\mathrm{X}$ \\
\hline & Moenkhausia agnesae GØy, 1965 & 14 & $41.7-3.5(33.8-50.5)$ & & $\mathrm{X}$ & $\mathrm{X}$ & \\
\hline & Moenkhausia collettii (Steindachner, 1882) & 20 & $39.5-2.8(32.2-48.1)$ & $\mathrm{X}$ & $\mathrm{X}$ & & $\mathrm{X}$ \\
\hline & Moenkhausia lepidura (Kner, 1859) & 35 & $45.6-3.7(34.0-44.9)$ & $\mathrm{X}$ & $\mathrm{X}$ & $\mathrm{X}$ & $\mathrm{X}$ \\
\hline & Tetragonopterus chalceus Spix y Agassiz 1829 & 14 & $60.4-7.9(44.1-86.9)$ & & $\mathrm{X}$ & $\mathrm{X}$ & $\mathrm{X}$ \\
\hline \multirow[t]{5}{*}{ Curimatidae } & Caenotropus labyrinthycus (Kner, 1858) & 14 & $77.2-9.6(59.0-102.8)$ & & & $\mathrm{X}$ & $\mathrm{X}$ \\
\hline & Chilodus punctatus Muller y Troschel, 1844 & 21 & $45.1-4.6(35.1-57.2)$ & $\mathrm{X}$ & $\mathrm{X}$ & & \\
\hline & Cyphocharax festivus Vari, 1992 & 8 & $64.5-3.6(54.1-71.0)$ & & & $\mathrm{X}$ & $\mathrm{X}$ \\
\hline & Cyphocharax spilurus (G nther, 1864) & 51 & $52.6-5.5(40.0-87.9)$ & $\mathrm{X}$ & $\mathrm{X}$ & $\mathrm{X}$ & $\mathrm{X}$ \\
\hline & Steindachnerina pupula Vari, 1991 & 17 & $68.8-3.5(49.2-71.1)$ & & & $\mathrm{X}$ & $\mathrm{X}$ \\
\hline Erithrinidae & Hoplias malabaricus (Bloch, 1794) & 5 & $67.8-8.3(52.5-97.4)$ & $\mathrm{X}$ & $\mathrm{X}$ & $\mathrm{X}$ & \\
\hline \multirow[t]{2}{*}{ Hemiodontidae } & Hemiodopsis semitaeniatus Kner, 1858 & 8 & $66.0-4.9(55.8-74.6)$ & & & $\mathrm{X}$ & $\mathrm{X}$ \\
\hline & Hemiodus unimaculatus (Bloch, 1794) & 6 & $71.2-4.9(62.9-84.0)$ & & & $\mathrm{X}$ & $\mathrm{X}$ \\
\hline Serrasalmidae & Serrasalmus striolatus Steindachner, 1908 & 8 & $153.2-6.9(138.2-165.5)$ & $\mathrm{X}$ & $\mathrm{X}$ & & \\
\hline Sternopygidae & Eigenmannia virescens (Valenciennes, 1842) & 19 & $146.8-17.9(95.1-230.2)$ & $\mathrm{X}$ & $\mathrm{X}$ & $\mathrm{X}$ & $\mathrm{X}$ \\
\hline \multirow[t]{2}{*}{ Cichlidae } & Apistogramma ramirezi Myers y Harry, 1948 & 16 & $28.2-2.3(23.9-34.1)$ & $\mathrm{X}$ & $\mathrm{X}$ & & \\
\hline & Mesonauta festivus Heckel, 1840 & 15 & $51.2-9.3(34.2-74.3)$ & $X$ & $\mathrm{X}$ & & \\
\hline Auchenipteridae & Tatia reticulata Mees, 1974 & 15 & $29.1-1.7(25.6-32.3)$ & & $\mathrm{X}$ & & $\mathrm{X}$ \\
\hline Pimelodidae & Pimelodella cristata (M ller y Troschel, 1848) & 17 & $63.2-7.0(46.2-78.0)$ & & & $\mathrm{X}$ & $\mathrm{X}$ \\
\hline
\end{tabular}

AL: Alto Yucao-Epoca de Lluvias, AS: Alto Yucao-Epoca de Sequ a, BL: Bajo Yucao-Epoca de Lluvias, BS: Bajo Yucao-Epoca de Sequ a 
Características del tracto digestivo y hábitos alimentarios de peces colombianos: SÁNCHEZ, R. ET AL.

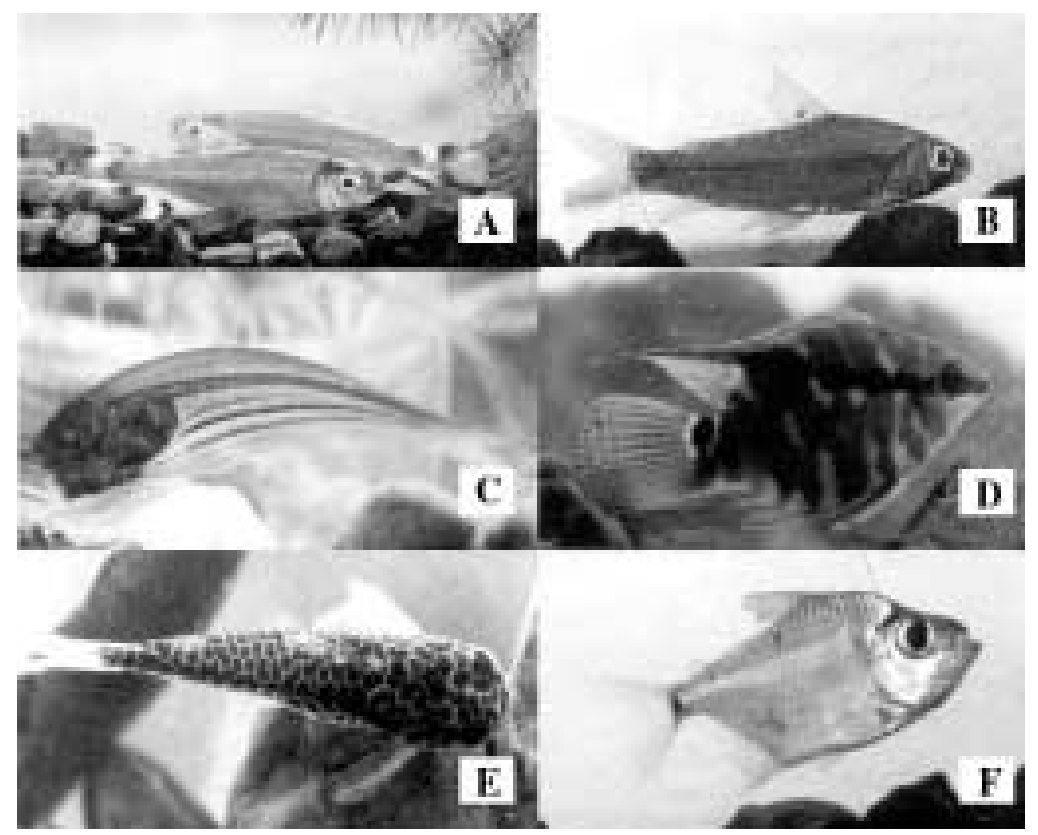

FIgURA 2. Morfología corporal de algunas especies analizadas. A. Bryconops melanurus, B. Steindachnerina pupula, C. Eigenmannia virescens, D. Mesonauta festivus, E. Tatia reticulata, F. Tetragonopterus chalceus.

Figure 2. Body morphology of some species analysed. A. Bryconops melanurus, B. Steindachnerina pupula, C. Eigenmannia virescens, D. Mesonauta festivus, E. Tatia reticulata, F. Tetragonopterus chalceus.
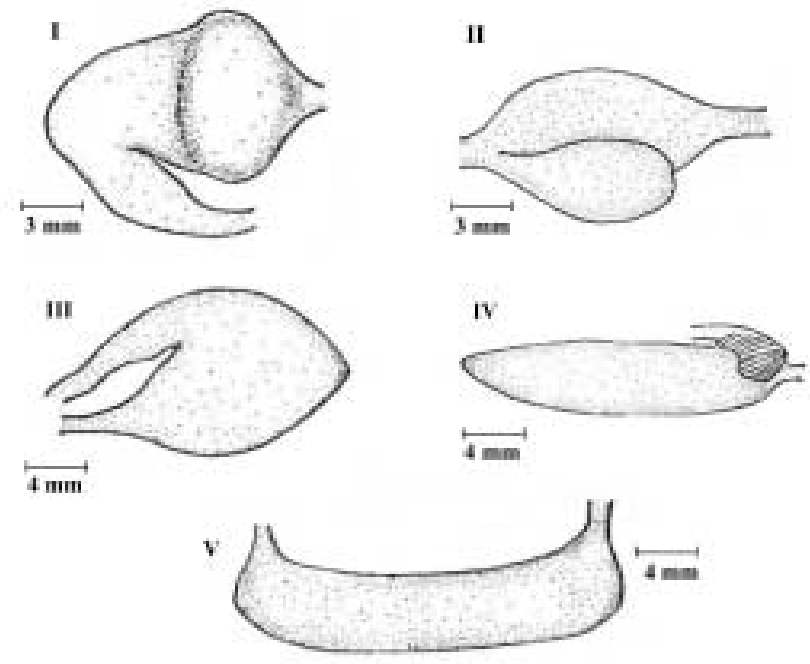

FiguRA 3. Formas del estómago I. Estómago en forma de bolsa asimétrica, con poco lumen y muy rígido (e.g. Cyphocharax spilurus). II. Estómago bilobado (e.g. Apistogramma ramirezi). III. Estómago en forma de bolsa (e.g. Bryconops melanurus). IV . Estómago alargado (Hoplias malabaricus). V. Estómago oblongo (e.g. Hemiodus unimaculatus)

FIGURE 3. Stomach shapes I. Asymmetric bag, small lumen and very rigid (e.g. Cyphocharax spilurus). II. Bilobate stomach (e.g. Apistogramma ramirezi). III. Bag shape stomach (e.g. Bryconops melanurus). IV . Elongated stomach (Hoplias malabaricus). V. Oblong stomach (e.g. Hemiodus unimaculatus) 
Gayana 67(1), 2003

Apistogramma ramirezi, Mesonauta festivus) y 24 para $H$. unimaculatus. Por otro lado, la relación entre longitud del intestino y la longitud estándar (LI/LE) se encontró entre 0,29 y 12,1 (Tabla II).

TABLA II. Características de las estructuras del tracto digestivo analizadas en las diferentes especies.

TABLE II. Characteristics of structures of digestive tract analysed in each species.

\begin{tabular}{|c|c|c|c|c|c|c|c|}
\hline & LEF II & Eili & $\begin{array}{l}\text { HAli } \\
\text { Hith. }\end{array}$ & MEy & $\begin{array}{l}\text { HELH } \\
\text { IFTHIN }\end{array}$ & HIDis & $\frac{H 1}{4}$ \\
\hline I & Mrnequelesm & $\mathbf{T}$ & $F=-1$ & 11: & Ate & He & $12=1 ;$ \\
\hline 1 & Alatsh & 5 & $\operatorname{Er},-12$ & D & DE. & + & LHa \\
\hline 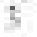 & No: & $T$ & $7=1-1=$ & 12 & 15: & $H$ & $1+1$ \\
\hline+ & Hothunger & 1 & Dind-1a & 17 & Dets & II & rate \\
\hline \pm & Hotharlin & 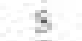 & $T=-12$ & II & Rts & 7 & $E=1$ \\
\hline 4 & Ihotumbitre & T & $2+12-74$ & 2 & Dty & 4 & Het \\
\hline 7 & 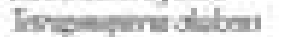 & T & 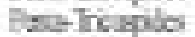 & 2 & Nut & -1 & $\mathrm{E}=1$ \\
\hline 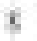 & 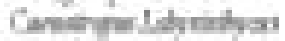 & 5 & whes & 21 & Dres & 7 & $F=12$ \\
\hline 1 & Oelspartas & 1 & Habi & 2 & Hos. & HD & $H=1$ \\
\hline 17 & rintulingen & 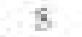 & nst: & 7 & Banden & ME: & $7 \pm 1$ \\
\hline 11 & inturatura & 3 & Ron & 5 & Devaris & 11 & 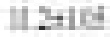 \\
\hline 15 & 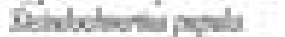 & 5 & Harly & I & Nobinatia & 2H & $7=1$ \\
\hline 11 & 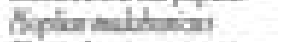 & 1 & Enes & Hi & $1+1$ & 4 & $1+1=$ \\
\hline 1 & 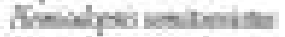 & $=$ & Grent & -1 & Dto. & 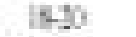 & $1=I$ \\
\hline 15 & Meotiuminth & 9 & Friem & 불 & MHos & n.1 & $11=-\pi$ \\
\hline H & Breularbohe & T & dintres & 1 & NE & EL & HEI \\
\hline$\Gamma$ & Doneuna & E & FH= & + & Et & MII & $7 \pm 11$ \\
\hline 11 & than man= & 1 & Clanes. & 11 & Eltad & Anin & $1 H=11$ \\
\hline It & 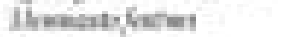 & $\mathbf{T}$ & Eings & HII & $12+1$ & Ahe & Fen \\
\hline 11 & Irintinit & 3 & E-E & $=1$ & 5U & nesis & $-1+1$ \\
\hline 1 & Halletumed & 5 & risery & Ly & Etw & Hers & $E=1$ \\
\hline
\end{tabular}

\section{COMPOSICIÓN DE LA DIETA}

La dieta fue muy variable entre las diferentes especies (Tabla III). Se registraron microalgas, plantas vasculares, frutos, semillas, insectos acuáticos y terrestres, larvas de insectos, otros invertebrados y peces. De acuerdo a la procedencia y características del alimento encontrado en el tracto digestivo, en una primera aproximación las especies se catalogaron como: 1 . detritívoros, a los que se les registró gran cantidad de material del fondo del río (p.e. C. spilurus) (Figura 4a); 2. herbívoros, a los que se les encontró mayor cantidad de componente vegetal tanto algas filamentosas como plantas superiores (p.e. M. festivus) (Figura 4b); 3. consumidores de perifiton (perifitófagos), cuyos conteni- dos estomacales se caracterizaron principalmente por presentar microalgas y microinvertebrados que hacen parte del perifiton (p.e. Chilodus punctatus)(Figura 4c) y 4. omnívoros, en los que indistintamente se encontró material vegetal como animal de origen diferente (p.e. B. melanurus) (Figura 4d). Serrasalmus striolatus fue la única especie en que se encontraron restos de peces en sus estómagos, junto con otros ítemes como frutos e invertebrados. Por otro lado, la dieta de Eigenmania virescens estuvo conformada sólo por ítemes de origen animal, por lo que se puede pensar que es un pez básicamente carnívoro (Figura 4e). En los estómagos de Hoplias malabaricus no se encontraron contenidos, por lo que esta especie no se tuvo en cuenta para los posteriores análisis. 
Características del tracto digestivo y hábitos alimentarios de peces colombianos: SÁNCHEZ, R. ET AL.

TABLA III. Presencia de ítemes alimentarios en la dieta de las especies analizadas.

TABLE III. Presence of items in the diet of analysed species.

\begin{tabular}{|c|c|c|c|c|c|c|c|c|c|c|c|c|c|c|c|c|c|c|c|c|}
\hline & & & & & & & & & & 11 & at & & & & & & & & & \\
\hline 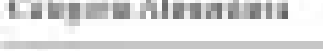 & 1 & 4 & 1 & 1 & + & 11 & 4 & 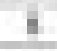 & 4 & III & 1 & 11 & II & it & H & IT & In & 19 & 1 & 1 \\
\hline 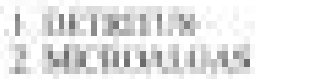 & & & & & & & & & & $\mathrm{K}$ & $\mathrm{H}$ & X & & & & & & & & \\
\hline 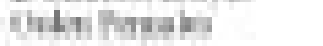 & & & & & & & & $\mathbf{H}$ & $\mathbf{H}$ & $H$ & $\mathbf{H}$ & H & H & 1 & & & & 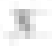 & & \\
\hline Mhidythir & & & & & & & & $x$ & $\mathbf{x}$ & $x$ & $\mathrm{x}$ & $\mathrm{x}$ & & & & & & & & \\
\hline ifturifirimat & & & & & & & & 1 & $\mathbf{t}$ & H & $\mathbf{A}$ & H & & & & & & & & \\
\hline Hithilitht & & & & & & & & 1 & & $\mathbf{H}$ & $a^{2}$ & H & & & & & & & & \\
\hline 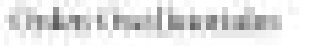 & & & $\mathrm{T}$ & $\mathrm{h}$ & I & & $x$ & & 1 & $\mathbf{k}$ & 1 & $\mathrm{x}$ & L & $x$ & & & II & 4 & & \\
\hline 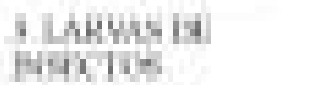 & & & & & & & & & & & & & & & & & & & & \\
\hline Whe Hquat & $x$ & & & & & $\mathrm{H}$ & & & & & & & & & & $\mathrm{x}$ & & & & \\
\hline 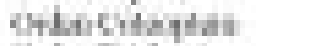 & A & & & & & & $y$ & & & & & & & & & $x$ & 1 & & & \\
\hline 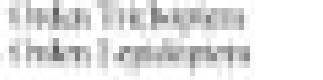 & & 4 & & 4 & & & A & & & & & & & & & & I & & & H \\
\hline A hrour. & & & & & & & & & & & & & & & & & & & & \\
\hline 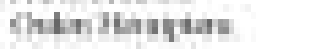 & & & $x$ & & $x$ & $x$ & $y$ & & & & & & & & 1 & & & & $\mathbf{H}$ & 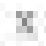 \\
\hline Holingivitin & 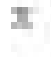 & 4 & & & & & & & & & & & & & & 1 & $\mathbf{T}$ & & $x$ & \\
\hline 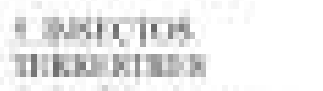 & & & & & & & & & & & & & & & & & & & & \\
\hline Anthintad: & & & & & $x$ & & & & & & & & & & & & & & $x$ & \\
\hline 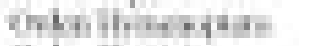 & 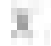 & & & & & & & & & & & & & & & & & & 4 & \\
\hline Whinimina: & & & & 4 & & $\mathbf{x}$ & & & & & & & & & & & & & & A \\
\hline Thloilherptu & & & & 4 & & & & & & & & & & & & & $\mathbf{x}$ & & & $x$ \\
\hline in wh hritm. & & & & & & & & & & & & & & & $\mathrm{T}$ & & & & & \\
\hline $\begin{array}{l}9 \text { iluint } \\
\text { milliphth }\end{array}$ & & $\mathrm{x}$ & & A & $x$ & $\mathrm{~A}$ & n & & & & & & & & & I & & & $\mathrm{X}$ & A \\
\hline 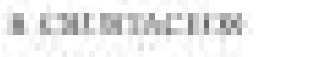 & & & & & & & & & n & & & & & & 1 & 1 & 1 & & & \\
\hline 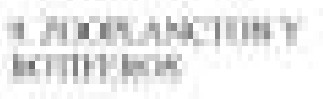 & & & & & & & & & H & $\mathrm{H}$ & & & h & & & & & & & \\
\hline It I in fling & & & & & & & & & & & & & & & & & & 4 & & \\
\hline II Hin & & & & & & & & & & & & & & & 1 & & & & & \\
\hline $\begin{array}{l}\text { lathotit } \\
\text { aymint }\end{array}$ & & & & & & & & & & & & & & & & & & & & \\
\hline Inimith haser & 1 & & & & & & & & & & & & & & 11 & & & & & \\
\hline 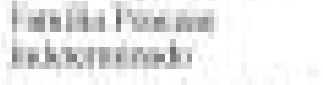 & & & 1 & H & 1 & H & 4 & & & & & & & H & & & I & H & 1 & H \\
\hline in inher thim & & & & 4 & $x$ & $\mathrm{~A}$ & a & & & $x$ & & & 4 & & & & & 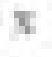 & $x$ & \\
\hline HIXH4hy & & H. & & & & & & & & & & & & & & & & & & $\mathbf{H}$ \\
\hline
\end{tabular}



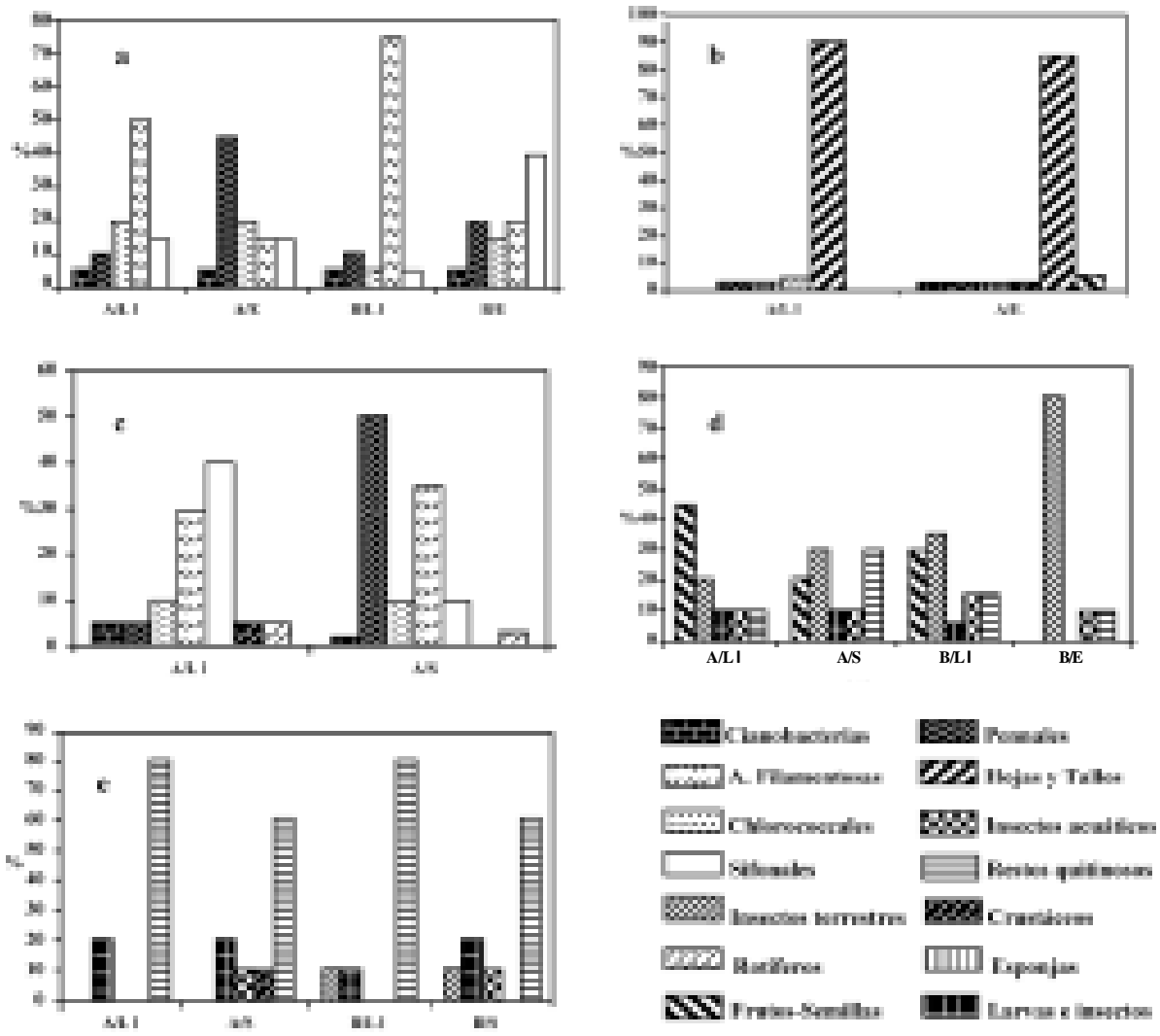

FIGURA 4. Variación temporal y espacial en los contenidos estomacales de peces del río Yucao. a. Cyphocharax spilurus, detritívoro. b. Mesonauta festivus, herbívoro. c. Chilodus punctatus, perifívoro. d. Bryconops melanurus, omnívoro. e. Eigenmmania virescens, carnívoro. A: Sector alto del río. B: Sector bajo del río. Ll: periodo más lluvioso. S: periodo menos lluvioso.

FIGURE 4. Temporal and spatial variation in the stomach contents of Yucao river fishes. a. Cyphocharax spilurus, detritívorous b. Mesonauta festivus, herbivorous. c. Chilodus punctatus, periphytivorous. d. Bryconops melanurus, omnivorous. e. Eigenmmania virescens, carnivorous. A: Highs Yucao. B: Down Yucao. Ll: rainiest period. S: less raining period.

\section{ANÁLISIS DECORRESPONDENCIA}

Con el análisis de correspondencia que analiza los grupos tróficos determinados a priori (Figura 5) se observó que en el componente 1 se disgregan tres grupos (Eigenvalue: 0.971): uno conformado por especies que se alimentan indistintamente de material animal y vegetal (omnívoros); otro por peces que se alimentan de plantas superiores y microalgas (herbívoros + perifitófagos); y un tercer grupo conformado por las especies que se alimentan de detritus. Con respecto al componente 2 (Eigenvalue 0,350 ) se separan las especies que se alimentan de plantas superiores de las especies que se alimentan de microalgas. Este análisis confirma la propuesta de los cuatro grupos tróficos propuestos.

Dentro del grupo de los omnívoros se puede discernir subgrupos separados fundamentalmente por el componente 2. Por ejemplo, Bryconops melanurus, Tetragonopterus chalceus, Tatia reticulata y Moenkhausia lepidura se agrupan por consumir fundamentalmente insectos. Otro subgrupo conformado por Pimellodella cristata y Tatia reticulata, que aparte de consumir ítemes similares a las especies anteriores, también consumen escamas (Tabla II). Todos los grupos sugeridos por el análisis de correspondencia coinciden con las composiciones de especies definidas a priori. 


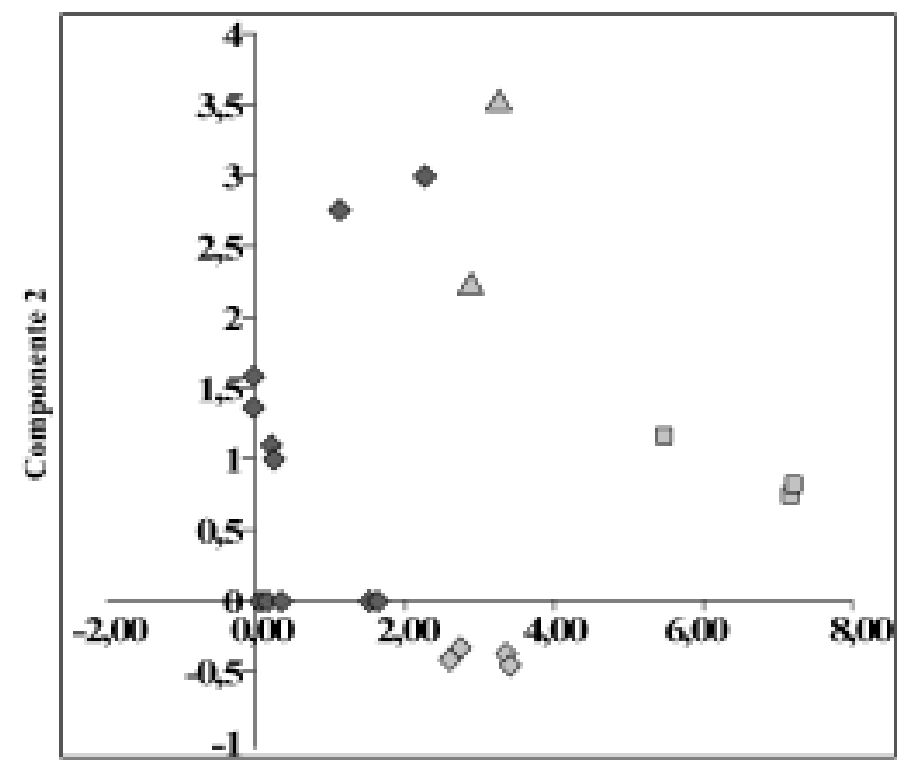

Componente 1

- Omnivores $\square$ Detrithores $\Delta$ Herbivoros $\diamond$ Perifitofagos

FIGURA 5. Ordenamiento de tipos tróficos de peces del río Yucao, obtenido a partir del análisis DECORANA.

FIGURE 5. Plotting of trophic types of fishes from river Yucao, obtained from detrended correspondence analysis.

Respecto al análisis de la composición dietaria y las diferentes morfologías del tracto digestivo, se encontró que la morfología de los dientes y del estómago son las características que mejor se relacionan con ciertos ítemes alimentarios (Figs. 6 y 7). Estas correspondencias concuerdan además con los grupos tróficos entregados por el primer análisis de correspondencia. La forma de los dientes (Fig. 6) se ordenó a lo largo del componente 1 asociado con tres tipos de dieta, las cuales son básicamente las mismas obtenidas en la Fig. 5. Un primer grupo de especies presentan dientes de variada forma, pero que tienen en común una dieta omnívora. Este grupo incluye los tipos de dientes DI-1, DI-2, DI-6, DI-5 y DI-8. Aunque no conforman un grupo discreto, se ubican luego especies herbívoras y perifitófagas, con dientes de tipo incisivos (DI-4), bilobados (DI-3) y cónicos (DI-7). Finalmente en el componente 1 se disgregan las especies carentes de dientes (DI-0) y asociadas a una dieta detritívora. En el componente 2 en cambio se pudo distinguir, por una parte, la separación de dientes asimétricos (DI-5) asociados al consumo de arácnidos y peces (ítemes 6 y 11), y por otra, a dientes de tipo incisivo (DI-4) relacionados con el consumo de esponjas. La forma del estómago (Fig. 7) se asoció en el componente 1 a un ordenamiento similar al de los grupos tróficos ya definidos. Por una parte el estómago de tipo bolsa (E-III) se corresponde con una dieta fundamentalmente omnívora, mientras que los estómagos de forma bilobada (E-II) y oblonga (E-V) son de especies herbívoras y perifitófagas. Por último se separan las especies con estómago de forma de bolsa asimétrica (E-I), lo que se corresponde con el consumo de detritus.

A pesar de no mostrar una concordancia clara con todos los grupos tróficos, otros atributos del tracto digestivo mostraron asociación a ciertos tipos de dieta. Por ejemplo, una mayor longitud del intestino se corresponde en el componente 1 con una dieta fundamentalmente de tipo detri-tívora (Eigenvalue: 0,630). 
Gayana 67(1), 2003

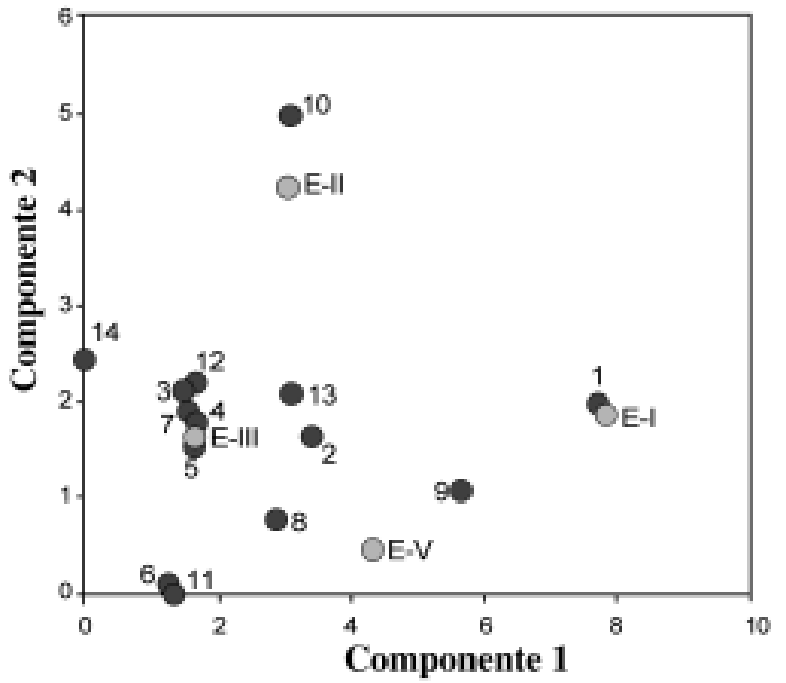

Figura 6. Ordenamiento de los diferentes ítemes alimentarios (en negro) y tipos morfológicos de estómagos (gris), a partir del análisis DECORANA. Los números de tipos de presa corresponden a los de la Tabla III y los códigos numéricos de los estómagos corresponden a los de la Figura 3.

FIGURE 6. Plotting of dietary items (black) and teeth morphological types (grey) obtained from detrended correspondence analysis. Item numbers are the same of the Table III and number codes of stomachs are explained in Fig.3.

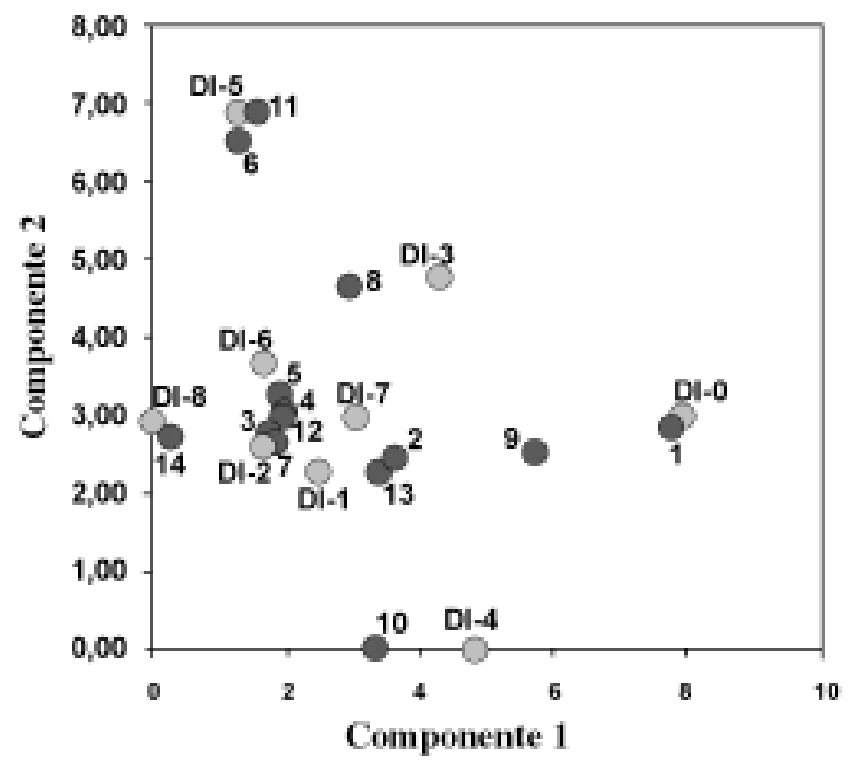

FigURA 7. Ordenamiento de los diferentes ítemes alimentarios (en negro) y tipos morfológicos de dientes (gris), a partir del análisis DECORANA. Los números de tipos de presa corresponden a los de la Tabla III. DI-0: Ausentes; DI-1: Pentacúspides; DI-2: Tricúspides; DI-3: Bilobados; DI-4: Incisivos; DI-5:Asimétricos; DI-6:Filiformes; DI-7:Cónicos; DI-8:Unicúspides.

FIGURE 7. Plotting of dietary items (black) and teeth morphological types (grey) obtained from detrended correspondence analysis. Item numbers are the same of the Table III. DI-0: Absent teeth; DI-1: Pentacuspid; DI-2: Tricuspid; DI3: Bilobates; DI-4: Incisives; DI-5: Asymmetrics; DI-6: Filiforms; DI-7: Conics; DI-8:Unicuspids. 


\section{DISCUSION}

Varios estudios han demostrado la relación existente entre la forma y características del tracto digestivo con los hábitos alimentarios. Para peces continentales, los trabajos de Hugueny \& Pouilly (1999) en el este de Africa, Xie et al. (2001) en China, Delariva \& Agostinho (2001) y Albrecht et al. (2001) en América del Sur, entre otros, han enfatizado estas relaciones no sólo entre especies de diferentes géneros sino entre especies cercanas. Todos estos resultados conllevan a determinar la gran influencia morfológica que presenta cada una de la especies para seleccionar $\mathrm{y}$ aprovechar los recursos disponibles.

A pesar de encontrar variabilidad de hábitos alimentarios en las especies de peces del río Yucao, se puede corresponder las características de algunas estructuras del tracto digestivo, que intervienen en la captura y asimilación del alimento, con cuatro conductas alimentarias o gremios tróficos generales: las especies detritívoras o sedimentófagas presentan boca subterminal y pequeña, lo que les permite succionar los fondos blandos tomando bocanadas de sedimento, dentición ausente, braquispinas papilosas y poco numerosas, estómago reducido y una molleja con pared muscularizada. Esto último les permite triturar mediante granos de arena el material orgánico, a lo que se suma un intestino muy largo. Estas características le permiten a estos organismos optimizar la deglución y absorción de materiales detríticos y elementos orgánicos poco particulados. Dentro de este grupo se encuentran las especies de los géneros Cyphocharax y Steindachnerina. Este tipo de hábito alimentario también es compartido por otros grupos de peces, como es el caso de la mayoría de los loricáridos que presentan gran diversidad en este sistema y en general en el Neotrópico (Burgess 1989; Sánchez et al. 1999; Delariva \& Agostinho 2001), manifestando la relevancia de este tipo de alimentación en sistemas poco productivos.

Como consumidores de perifiton se enmarcan Chilodus punctatus y Caenotropus labyrinthycus, alimentándose principalmente de microalgas. Se caracterizan por presentar una boca terminal o subterminal con labios flexibles y dientes pequeños. Los dientes son incisivos o bilobados, que les ayudan a raspar los fondos duros. Tienen un estómago oblongo que es rígido, con un intestino casi dos veces su longitud estándar y un número intermedio, respecto a las otras especies, de ciegos pilóricos.
Los peces herbívoros se caracterizan por tener una boca terminal amplia y una serie de dientes incisivos o cónicos que les permiten cortar las estructuras de su alimento. Entre éstos tenemos a Hemiodopsis semitaeniatus, Hemiodus unimaculatus y Mesonauta festivus; sin embargo, esta última especie presenta un estómago bilobado diferente al oblongo que presentan las otras dos.

A los peces que presentaron diversidad en el origen de los contenidos estomacales se les catalogó como omnívoros, se caracterizan por tener una boca terminal o subterminal amplia. Estos tienen dientes fuertes unicúspides, multicúspides o asimétricos, utilizados para triturar el alimento, el estómago tiene forma de bolsa, es elástico y consistente. La longitud del intestino es variable, al igual que el número y tamaño de las braquispinas. Dadas estas características, la variabilidad morfológica en este grupo es importante y puede estar relacionada con el tipo de recurso que explota cada especie, aunque como se ve en la variación temporal y espacial de los contenidos estomacales, los organismos consumen lo que hay disponible y no son muy selectivos. Sin embargo, algunas especies tienen tendencia a consumir mayor cantidad de material vegetal que animal y la variabilidad morfológica en ciertos órganos o tejidos, como la boca y los dientes, sería un reflejo de las preferencias alimentarias de cada especie. Por ejemplo, Apistogramma ramirezi y Hemigrammus barrigonae consumen mayor proporción de alimento vegetal, mientras Serrasalmus striolatus presenta mayor consumo de ítemes animales. Por otro lado, para Eigenmmania virescens sólo se encontró en su dieta material de origen animal (larvas de insectos, restos de crustáceos). Esta especie presenta un estómago en forma de bolsa pero sus dientes son filiformes y por lo tanto no presentaría concordancia con el patrón de las especies catalogadas en este grupo. Asimismo, Tatia reticulata y Pimelodella cristata, aunque presentan estómagos en forma de bolsa, la forma de los dientes es diferente. Por lo tanto, una revisión detallada de la dieta entre estas especies nos puede dilucidar relaciones más estrechas entre sus características morfológicas y el tipo de alimento que consumen.

Aunque en ninguno de los individuos de Hoplias malabaricus se encontró contenido en su estómago, es una especie considerada piscívora, debido a que presenta características del tracto digestivo similares a otras definidas dentro de este gremio alimentario, como p.e. Acestrorhynchus 
falcirostris Curvier 1819 (Castro \& Sánchez 1994). Tiene boca terminal, amplia y fuerte, conformada por dientes caninos, su estómago es largo y su intestino corto, características relevantes en especies con este tipo de alimentación.

En términos generales, existe un patrón en cuanto a la relación entre los hábitos alimentarios de los peces del río Yucao (detritívoro, consumidor de perifiton, herbívoro, omnívoro) y la morfología del tracto digestivo, siendo la forma del estómago y los dientes las estructuras que más se relacionan con cada grupo trófico.

\section{AGRADECIMIENTOS}

Los autores agradecen a la Universidad Nacional de Colombia, al Instituto de Ciencias Naturales, al Instituto Colombiano para el Desarrollo de la Ciencia y Tecnología-COLCIENCIAS y al Depto. de Zoología de la Universidad de Concepción, por el apoyo logístico brindado. Al Dr. Gabriel Guillot, a William Castro y Alfredo Saldaña, por su colaboración en la realización de este trabajo. Esta publicación es parte de la formación doctoral del primer autor en el programa de Ciencias Biológicas -Area Zoología, de la Universidad de Concepción. Estudio financiado particularmente por el proyecto 200.113.054-1.3 de la Dirección de Investigación de la Universidad de Concepción.

\section{BIBLIOGRAFIA}

Albrecht, M.P., M.F.N. Ferreira \& E.P. CARAmaschi. 2001. Anatomical features and histology of the digestive tract of two related neotropical omnivorous fishes (Characiformes; Anostomidae). Journal of Fish Biology 58:419-430

BuRgESs, W. 1989. An atlas of freshwater and marine CATFISHES. A preliminary survey of the siluriformes. T.F.H. Publications, Inc. New Jersey USA.784 pp.

CAmargo, Z.M. 1992. Contribución al conocimiento de la fauna íctica de Caño Limón (Arauca) durante un ciclo anual de lluvias y sequía. Tesis Universidad Nacional de Colombia, Bogotá. 80 pp.

CASTRO, W. \& R.M. SÁnchEZ. 1994. Estudio íctico comparativo entre dos ambientes del río Yucao, Departa- mento del Meta. Tesis Universidad Nacional de Colombia, Bogotá. $136 \mathrm{pp}$

Delariva, R.L. \& A.A. Agostinho. 2001. Relationship between morphology and diets of six neotropical loricariids. Journal of Fish Biology 58:832-847

Forster, V. K. 1969. Amazonische desmidieen. Amazoniana II (1/2):5-537

Hugueny, B. y M. PoullLy. 1999. Morphological correlates of diet in an assemblage of West African freshwater fishes. Journal of Fish Biology 54:1310-1325

JAKSIC, F. 2001. Ecología de comunidades. Ediciones Universidad Católica de Chile. 233pp.

LAGLER, K.F., J.E. BARDACH, R.R. MilLER \& D.R. MAY. 1977. Ichthyology: The study of fishes. John Wiley \& Sons.506 pp.

LIEM, K.F. 1980. Acquisition of energy by teleosts: Adaptative mechanisms and evolutionary patterns. En ALI, M.A. (Ed). Environmental Physiology of fishes. Plenum Press, New York. 299-334.

Lowe-McConNELL, R.H. 1987. Ecological studies in tropical fish communities. Cambridge University Press. London.176 pp.

Needham, J. y P. NeEdham. 1982. Guía para el estudio de los seres vivos de las aguas dulces. Editorial Reverte, S.A. España.131 pp.

Nelson, J. 1994. Fishes of the World. John Wiley \& Sons, New York. 600 pp

Nikolsky, G.V. 1963. The Ecology of Fishes. Academic press. London. 352 pp.

Pennak, R. W., 1978. Fresh-Water invertebrates of United States. John Wiley \& Sons, Inc.USA, 803 pp.

Press, J. A. y G. Colomine. 1981. Métodos para el estudio de los alimentos y las relaciones tróficas de los peces. Universidad Central de Venezuela, Caracas. $129 \mathrm{pp}$.

Roldan, G. 1988. Guía para el estudio de macroinvertebrados acuáticos del departamento de Antioquia. Editorial Presencia Ltda. 217 pp.

SÁnchez, R.M., W. Castro \& G. Galvis. 1999. Similaridad de la composición íctica entre dos ambientes del río Yucao, sistema del río Meta. Revista de la Academia Colombiana de Ciencias exactas, Físicas y Naturales 23(Suplemento especial):567-574

Sioli, H. 1975. Amazon tributaries and drainage basins. Ecological Studies 10:199-213.

Uherkovich, G. y G.W. SchmidT. 1974. Phytoplankton taxa in dem zentralamazonische schewemmlandsee lago do Castanho. Amazoniana 5: 243-283.

WILSON, J.B. 2002. Golliwog: programa estadístico.

XIE, S., Y. CUI, y Z. LI. 2001. Dietary-morphological relationships of fishes in Liangzi Lake, China. Journal of Fish Biology 58: 1714-1729.

YACUBSON, S., 1969. Algas de ambientes acuáticos continentales, nuevas para Venezuela (Cyanophyta, Clorophyta). Editorial Universitaria de la Universidad de Zulia. $87 \mathrm{pp}$.

Fecha de recepción: 08/04/01

Fecha de aceptación: 01/03/03 